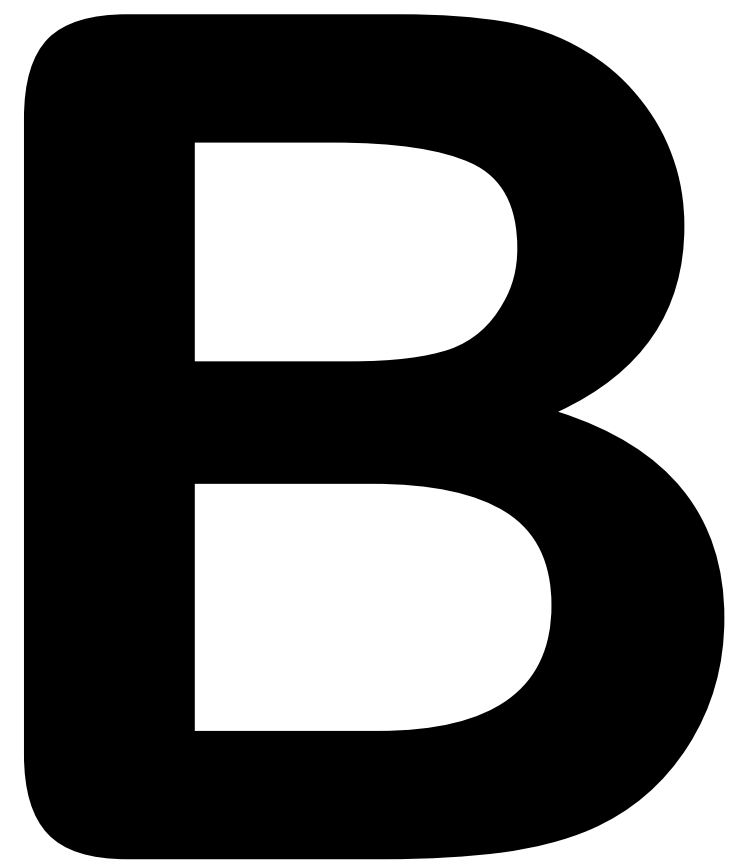

\title{
Clinical Trials in Radiology (CT)
}




\section{Wednesday, February 28}

10:30 - 12:00

Sky High Stage

Interventional Radiology

\section{CT 2}

\section{Clinical Trials in Radiology 1}

Moderators:

M. Dewey; Berlin/DE

R.L. Ehman; Rochester, MN/US

\section{B-0184 10:30}

A two-center phase III, randomised, controlled study of MRgFUS vs EBRT in patients with metastatic non-spinal bone disease: palliative strategy for cancer-induced bone pain

A. Napoli, R. Scipione, A. Leonardi, H.-P. Erasmus, S. Dababou,

C. Marrocchio, C. Catalano; Rome/IT (alessandro.napoli@uniroma1.it)

Purpose: Purpose: to examine the clinical outcome of MR-guided focused ultrasound (MRgFUS) and external-beam radiation therapy (EBRT) in patients with painful bone metastasis.

Ethics committee approval: This study received institutional ethics committee approval. All patients provided written informed consent.

Methods and Materials: Patients with solid tumors and one or more bone metastasis were included. Eligible patients were $\geq 18$ years of age, had radiologically-proven bone metastasis, could safely undergo both MRgFUS and radiotherapy and had pain scores $\geq 4$ (on 0 -to- 10 numeric rating scale). Participants were randomly assigned (1:1 ratio) to receive MRgFUS or EBRT. The primary endpoint was treatment response, defined as a reduction of $\geq 2$ points in worst pain by week-4, accompanied by a stable or reduced opioid dose, compared with baseline. Secondary endpoints assessed average pain, interference of pain with activities, breakthrough pain, mood, quality of life, and adverse events.

Results: 233 patients (M:125,F:108) were enrolled and randomly assigned to MRgFUS(116) and EBRT(117). In the MRgFUS arm, 90 patients $(77.6 \%)$ achieved the primary endpoint, compared with $92(78.6 \%)$ in the EBRT arm (adjusted odds ratio, $1.07 ; p=0.818$ ). There were no significant differences in average pain, pain interference with activity, breakthrough pain, mood and quality of life between arms.

Limitations: This work only considers short-term results, assessing primary and secondary outcomes within the first month after treatment.

Conclusion: The role of MRgFUS in cancer-induced bone pain is comparable to EBRT, with the advantage of radiation-free and single-session procedure. MRgFUS is limited to non-spinal locations.

Funding for this study: This study was conducted without any direct founding.

$10: 40$

Discussant

M. Szczerbo-Trojanowska; Lublin/PL

\section{B-0185 10:45}

Percutaneous microwave thermal ablation (MWA) for patients with malignant lung tumors: mid-term results of a prospective multicenter study (MALT study)

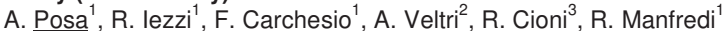

L. Bonomo ${ }^{1}{ }^{1}$ Rome $/ I T,{ }^{2}$ Orbassano $/ I T,{ }^{3}$ Pisa/IT (alessandro.posa@gmail.com)

Purpose: To assess the feasibility, safety, and efficacy of MWA in the treatment of unresectable primary (MALT1) and secondary (MALT2) pulmonary neoplasms.

Ethics committee approval: This study was approved by the Ethics Committee of our Institution and written informed consent was obtained for each patient prior to enrollment.

Methods and Materials: All patients with lung tumors up to $4 \mathrm{~cm}$ in size addressed to MWA by a multidisciplinary team were enrolled in the study. The lesions were classified by histotype (primary - MALT1, secondary - MALT2), site (hilar, parenchymal, or subpleural) and size $(<2,2-3,3-4 \mathrm{~cm})$. A standardized treatment was used for all lesions, based on histotype and size $(30 \mathrm{~W}, 50 \mathrm{~W}$ and $70 \mathrm{~W}$ and $40 \mathrm{~W}, 60 \mathrm{~W}$ and $80 \mathrm{~W}$, for primary and secondary tumors, respectively). The primary endpoint was complete response (CR) determined using enhanced CT scan, performed at 1, 3, 6, 12, and 18 months. Secondary endpoints were technical efficacy, incidence and grade of adverse events (AEs) as well as relationship between power applied and necrosis volume (power-efficacy correlation) evaluated on 1-month CT follow-up.
Results: Forty-four MWAs were performed on 35 patients (24 men; 11 women). Technical success was obtained in $100 \%$ of cases. No major complications as well as procedural death were recorded; $45.4 \%$ of minor complications occurred, $40.9 \%$ of them not requiring any treatment. A direct relationship between power applied and necrosis volume was obtained in all procedures. The 6 -months CR rates were of $100 \%$ and $92.3 \%$ in the MALT1 and MALT2 group respectively.

Limitations: The most important limitation of this study is represented by the limited number of patients enrolled, which can affect the values of response rates as well as complication rates.

Conclusion: Percutaneous CT-guided MWA seems to be a safe, feasible, and effective treatment for unresectable malignant lung tumors up to $4 \mathrm{~cm}$ in size. Funding for this study: There was no funding for this study.

10:55

Discussant

F. Gleeson; Oxford/UK

\section{B-0186 11:00}

Use of electromagnetic navigation improves performances in CT-guided interventions: a multicentric, prospective and randomised clinical trial A. Mounier, R.-C. Rouchy, M. Medici, E. Chipon, A. Moreau-Gaudry, I. Bricault; Grenoble/FR (amounier1@chu-grenoble.fr)

Purpose: To evaluate the clinical benefit of using an electromagnetic navigation system during CT-guided interventional radiology procedures.

Ethics committee approval: French Sud-Est V ethical committee (12/06/2013)

Methods and Materials: This study included 455 patients between December 2013 and July 2015 across nine participating hospitals. After randomization, percutaneous procedures were carried out either under conventional CT guidance (CT group), or using the additional assistance of IMACTIS-CT® navigation system (NAV group). The primary outcome was evaluated by a blinded expert committee. It assessed the safety (number of serious adverse events), efficiency (number of targets reached) and performance (number of CT control acquisitions required to reach the target) of CT-guided interventions.

Results: All patients (227 patients in NAV group and 228 patients in CT group) were included in the intention-to-treat analysis. Missing data of 4 patients were re-coded according to a worst-case scheme in disfavor of navigation. In noninferiority analysis, NAV system was as safe (CI90 [-0.03;0.032]; $\Delta \mathrm{C}=0.07)$ and as efficient ( $\mathrm{Cl} 90$ [-0.03;0.008]; $-\Delta \mathrm{C}=-0.1)$ as conventional guidance. The number of CT control demonstrated to be reduced using NAV $(5[3 ; 9])$ vs CT (6[3;11]) $p=0.015$

Limitations: For 38 patients, the intervention was only partially achieved according to the initially randomized protocol: in most cases (33/38), radiologist went back to CT instead of NAV protocol, because of respiratory movements and/or lack of experience with the navigation system

Conclusion: The use of an electromagnetic navigation system allows to reach the targets with fewer CT control acquisitions than with conventional CT guidance.

Funding for this study: French Ministry of Health.

Author Disclosures:

I. Bricault: Advisory Board; member of the scientific advisory board of Imactis.

$11: 10$

Discussant

T. Penzkofer; Berlin/DE

\section{B-0187 11:15}

Randomised clinical trial of portal vein embolisation using n-butylcyanoacrylate vs polyvinyl alcohol particles plus coils for liver hypertrophy before major hepatectomies

J.H.M. Luz ${ }^{1}$, E. Coimbra' ${ }^{1}$, T. Bilhim ${ }^{1}$, F.V. Gomes ${ }^{1}$, N.V. Costa ${ }^{1}$, M.T. Correia ${ }^{1}$ P. Luz ${ }^{2}$, E. Barroso ${ }^{1} ;{ }^{1}$ Lisbon/PT, ${ }^{2}$ Rio de Janeiro/BR (jhugoluz@gmail.com)

Purpose: The aim of this study is to compare the efficacy of portal vein embolisation (PVE) using n-butyl-cyanoacrylate (NBCA) versus polyvinyl alcohol (PVA) particles plus coils in the induction of liver hypertrophy before major hepatectomies.

Ethics committee approval: The hospital review board and local ethics committee approved the study protocol.

Methods and Materials: This is a randomised clinical trial, single site. Eighty consecutive patients with resectable liver tumors and indication for preoperative PVE are randomised for one of two arms (40 patients in each arm). Primary endpoint is to compare the degree of hepatic hypertrophy, through computed tomography volumetry, at 14 and 28 days after PVE. Secondary endpoints are the comparative analysis of the two PVE methods (complications, cost, procedure time, contrast usage and radiation exposure) and post-operative outcome (accomplishment of the planned surgery, causes of hepatectomy suspension and liver failure incidence). 
Results: PVE with NBCA has shown a higher degree of hypertrophy (left lobe increase in size was $64 \pm 23 \%$ vs. $41 \pm 12$ with NBCA versus PVA plus coils). The amount of contrast medium used for the procedure was $111 \pm 34 \mathrm{ml}$ and $223 \pm 43 \mathrm{ml}$, for NBCA and PVA plus coils, respectively. The trial is ongoing (still recruiting).

Limitations: The trial is not multicentric.

Conclusion: PVE with NBCA seems more effective in promoting liver hypertrophy. The overall PVE cost, procedure time and contrast media use was substantially lower through the NBCA approach.

Funding for this study: There was no funding for this trial.

$11: 25$

Discussant

K.A. Hausegger; Klagenfurt/AT

\section{B-0189 11:30}

Comparing different thrombectomy techniques in five large-volume centres: a 'real world' observational study

A.C. Hesse ${ }^{1}$, A. Zapf ${ }^{1}$, J. Liman ${ }^{1}$, J. Fiehler ${ }^{2}$, A. Mpotsaris ${ }^{3}$, P. Schramm ${ }^{4}$, A. Berlis ${ }^{5}$, M. Knauth ${ }^{1}$, M.-N. Psychogios ${ }^{1} ;{ }^{1}$ Göttingen/DE, ${ }^{2} \mathrm{Hamburg} / D E$,

${ }^{3}$ Aachen/DE, ${ }^{4}$ Lübeck/DE, ${ }^{5}$ Augsburg/DE

(amelie.hesse@med.uni-goettingen.de)

Purpose: Thrombectomy has become the standard of care for acute ischaemic stroke due to large vessel occlusion. Aim of this study was to compare the radiological outcomes and time metrics of the various thrombectomy techniques.

Ethics committee approval: Approved by the ethics committee of the University Medical Centre Goettingen.

Methods and Materials: In this retrospective, multicentre study we analysed the data of 450 patients with occlusion of the anterior circulation, treated in five high-volume centres from 2013 to 2016. The treatment techniques were divided in three categories: first-pass use of a large-bore aspiration-catheter; first-pass use of a stent-retriever; and primary combined approach (PCA) of an aspirationcatheter and stent-retriever. Primary endpoints were successful reperfusion and groin to reperfusion time. Secondary endpoints were the number of attempts and occurrence of emboli in new territory (ENT). The primary analysis was based on the intention to treat groups (ITT).

Results: The ITT-analysis showed significantly higher reperfusion rates, with $86 \%$ of successful reperfusion in the PCA-group compared with $73 \%$ in the aspiration group and $65 \%$ in the stent-retriever group. There was no significant difference in groin to reperfusion time regarding the used technique. The secondary analysis showed an impact of the technique on the number of attempts and the occurrence of ENTs. Lowest ENT rates and attempts were reported with the combined approach.

Limitations: Limitations of our study include the retrospective, observational design and the non-standardised fashion of thrombectomy, as well as that $73 \%$ of the interventions were analysed by a core-laboratory, while a part of angiographic outcomes was self-reported by the individual centres.

Conclusion: The combined first-pass deployment of a stent-retriever and an aspiration-catheter was the most effective technique for reperfusion of anterior circulation large vessel occlusion. Our results correlate with the latest singlecentre studies, reporting very high reperfusion rates with PCA variations.

Funding for this study: There was no funding for this study.

Author Disclosures:

J. Fiehler: Consultant; Acandis, Bayer, Boehringer-Ingelheim, Codman,

Covidien, Medtronic, MicroVention, Penumbra, Philips, Sequent, Siemens,

Stryker. Equipment Support Recipient; Medtronic, MicroVention, DFG, EU,

BMBF, BMWi. M. Knauth: Consultant; Siemens, Penumbra, Stryker, Acandis,

Bayer. M. Psychogios: Consultant; Siemens, Penumbra, Stryker.

$11: 40$

Discussant

H.A. Deutschmann; Graz/AT

\section{B-0188 11:45}

Paclitaxel-coated balloon angioplasty for the treatment of symptomatic central venous stenosis in dialysis access: results from a prospective randomised control trial

P. Papadimatos, P. Kitrou, K. Katsanos, N. Christeas, D. Karnabatidis;

Patras/GR (pnpapadimatos@yahoo.gr)

Purpose: To investigate the safety and effectiveness of paclitaxel-coated balloons (PCB) for the treatment of dialysis access symptomatic central venous stenosis.

Ethics committee approval: The above mentioned trial has been approved by the ethics committee of the University of Patras.

Methods and Materials: Within 20 months (January 2014 to August 2015), 40 dialysis patients [19/40 arteriovenous fistulas (AVF) and 21/40 arteriovenous grafts (AVG)] with symptomatic central venous stenosis were randomised to undergo PCB [PCB group, $\mathrm{n}=20 ; 14 / 20 \mathrm{~m}$ ale; age: 56.7 (25-81)] or conventional balloon angioplasty [CBA group, $n=20$; 15/20 male; age: 57 (3381)]. There were $15 / 20$ restenotic lesions in PCB group and $12 / 20$ in CBA group. In 25/40 cases patients had an ipsilateral catheter insertion in the past. Primary endpoint was clinically assessed intervention-free period (IFP) of the treated segment at 6 months, while secondary endpoints included complication rates during follow-up period and subgroup analysis to identify factors influencing IFP.

Results: Mean follow-up period was 180 days (5-479). No complications were observed. Median IFP was significantly better in PCB group (PCB group: 179 days vs CBA group: 124.5 days, $p=0.026$; HR $0.445 \mathrm{Cl}$ : $0.217-0.909$ ). There was no significant difference between AVGs and AVFs $(p=0.17)$, treatment of de novo vs restenotic lesions $(p=0.33)$ or prior presence of catheter insertion $(p=0.21)$. In restenotic lesion of PCB group, longitudinal comparison between treatments also showed a significant difference in favour of PCB treatment (median IFP in $\mathrm{PCB}^{*}$ group 177 vs 91 days in $\mathrm{CBA}^{*}$ group, $\mathrm{p}=0.01$; HR 0.338 Cl: 0.148-0.774).

Limitations: The major limitations are that we are talking about a single center randomised control trial with no core lab support.

Conclusion: Paclitaxel-coated balloons had significantly better results compared to CBA for the treatment of symptomatic central venous stenosis in dialysis access in this randomised study. Longitudinal comparison of treatments in the same patients also showed a significant difference in favour of PCBs.

Funding for this study: No funding.

$11: 55$

Discussant

R. lezzi; Rome/IT 


\section{Thursday, March 1}

\section{$10: 30-12: 00$}

Sky High Stage

\section{Cardiac}

\section{CT 6}

\section{Clinical Trials in Radiology 2}

Moderators:

M. Dewey; Berlin/DE

R.L. Ehman; Rochester, MN/US

\section{B-0595 10:30}

MEDIRAD EARLY-HEART study: early clinical and biological predictors of radiotherapy-induced cardiac toxicity in breast cancer

M.-O. Bernier ${ }^{1}$, S. Jacob ${ }^{1}$, A.P.G. Crijns ${ }^{2}$, J.A. Langendijk ${ }^{2}$, R. Vliegenhart ${ }^{2}$,

S.E. Combs ${ }^{3}$, M. Mayinger ${ }^{3}$, A. Eraso ${ }^{4}$, F. Guedea ${ }^{5}$ M. Fiuza ${ }^{6}$, S. Constantino

Rosa Santos ${ }^{6}$, E. Mousseaux ${ }^{7}$, E. Cardis ${ }^{8}$, G. Frija ${ }^{7} ;{ }^{1}$ Fontenay-aux-Roses/FR

${ }^{2}$ Groningen/NL, ${ }^{3}$ Munich/DE, ${ }^{4}$ Girona/ES, ${ }^{5}$ L'Hospitalet del Llobregat/ES,

${ }^{6}$ Lisbon/PT, ${ }^{7}$ Paris/FR, ${ }^{8}$ Barcelona/ES (marie-odile.bernier@irsn.fr)

Purpose: Breast cancer $(\mathrm{BC})$ radiotherapy $(\mathrm{RT})$ leads to incidental radiation of the heart, resulting in increased risk of a variety of heart diseases. Identifying BC patients with the highest-risk of radiation-induced cardiac complications is crucial for developing strategies for primary and secondary prevention. Within the European MEDIRAD project (http://www.medirad-project.eu/), the multicentre prospective EARLY-HEART study aims to identify the most important cardiac imaging and circulating biomarkers of radiation-induced cardiovascular changes in the first 2 years after BC RT.

Ethics committee approval: Obtained in the 5 investigating centers.

Methods and Materials: This multicenter study will include 250 female BC patients aged 40-75 years treated with RT in 5 hospitals (France, Germany, Netherlands, Portugal, Spain). Compared with baseline cardiac imaging information evaluated before RT, 2D-speckle-tracking echocardiography and cardiac magnetic resonance imaging will assess changes in myocardial function and myocardial tissue anomalies respectively 6 to 24 months after RT, and computed tomography will assess anatomical changes in coronary arteries 24 months after RT. Blood samples will be collected before RT, at the end of $\mathrm{RT}$, at 6 and 24 months to identify circulating biomarkers changes. The relationships between dose distribution to the heart during RT and early cardiovascular changes will be analysed.

Results: Inclusions started in September 2017 and will provide first results in early 2019

Limitations: Only subclinical events are expected during follow-up.

Conclusion: It will identify biomarkers of early heart side effects and improve understanding of the mechanisms and circumstances that underlie their development.

Funding for this study: Funding: EU grant $n^{\circ} 755523$

10:40

Discussant

V.E. Sinitsyn; Moscow/RU

\section{B-0596 10:45}

Low-dose chest CT vs conventional chest x-ray prior to cardiac surgery: the CRICKET study

R.P.J. Budde ${ }^{1}$, A.M. den Harder ${ }^{2}$, T. Leiner ${ }^{2}$, P.A. de Jong ${ }^{2}$, J. Karady ${ }^{3}$,

C. Chun ${ }^{3}$, A. Bogers ${ }^{1}$, P. Horvat-Maurovich ${ }^{3}$, L. de Heer $^{2} ;{ }^{1}$ Rotterdam/NL,

${ }^{2}$ Utrecht/NL, ${ }^{3}$ Budapest/HU (r.budde@erasmusmc.nl)

Purpose: Embolic calcifications are the most important cause of postoperative strokes after cardiac surgery. Strokes can be caused by manipulation and clamping of the aorta during surgery. The aim of this study is to investigate if improved preoperative knowledge of aortic calcifications acquired with a lowdose chest CT can prevent postoperative strokes by optimising the surgical strategy.

Ethics committee approval: Ethics approval was obtained. All participants sign informed consent.

Methods and Materials: A prospective multicenter randomised controlled trial design is used. Inclusion criteria are patients scheduled for cardiac surgery aged 18 years or older. Exclusion criteria are emergency surgery, a recent chest/cardiac CT (past three months), unwillingness to be informed about unrequested findings, concomitant or prior participation in a study with radiation exposure and pregnancy. Patients $(n=1,724)$ are randomised between a control arm, which receives routine preoperative work-up including a chest $\mathrm{x}$-ray, and the intervention arm, which receives an additional non contrast-enhanced chest CT at a submillisievert radiation dose. Primary outcome is the postoperative in-hospital stroke rate. Secondary outcomes are altered surgical approach based on CT findings and cost-effectiveness.

Results: Currently 3 centers are participating and over 500 patients are included. As of mid 2017, in the largest including center 417 patients were randomised (207 in the control group and 210 in the intervention group of which $94 \%$ and $86 \%$ received the allocated work-up, respectively). In the combined 238 patients randomised to the CT group, mean radiation exposure was $0,64 \mathrm{mSv}$. Ventral calcifications in the aorta were seen in $23 \%$ of patients on CT.

Limitations: The secondary objective change in surgical approach is surgeondependent and therefore subjective.

Conclusion: The CRICKET study will determine if it is useful and costeffective to replace preoperative chest $x$-ray by low-dose chest CT. Funding for this study: ZonMW. Project 837001403

10:55

Discussant

J. Bremerich; Basle/CH

\section{B-0597 $11 \cdot 00$}

Computed tomography (CTA) vs invasive coronary angiography (ICA) in patients with atypical chest pain and suspected coronary artery disease (CAD): gender analysis of a randomised study

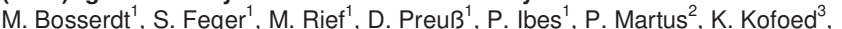
M. Laule ${ }^{1}$, M. Dewey'; ${ }^{1}$ Berlin/DE, ${ }^{2}$ Tübingen/DE, ${ }^{3}$ Copenhagen/DK

Purpose: To investigate if females equally benefit from performing CTA instead of ICA if they present with atypical chest pain and a clinical indication for ICA.

Ethics committee approval: By ethics committee of Charité (EA-1-080-08) and German Federal Office for Radiation Protection (Z5-22462/2-2008-048). All patients gave written informed consent.

Methods and Materials: This is gender subanalysis of single-center coronary artery disease management (CAD-Man) study including 329 patients.

Results: Median length of stay was equally reduced in CTA group for females (30.0 hours, interquartile range [IQR]: 3.3-78.3 vs. ICA group: 53.0 hours, IQR: 49.7-75.3; $P=0.002$ ) and males (30.0 hours, IQR: $3.9-71.3$ vs. ICA group: 53.0 hours, IQR: 49.1-76.8; $\mathrm{P}=0.005)$. Radiation dose was similar in CTA and ICA group both for females $(4.7 \mathrm{mSv}$, IQR: $3.4-6.6$ vs. $5.7 \mathrm{mSv}$, IQR: $3.0-9.2 \mathrm{mSv}$; $\mathrm{P}=0.41)$ and males (5.3 mSv, IQR: $4.7-10.6$ vs. $6.9 \mathrm{mSv}$, IQR: $4.4-12.7 \mathrm{mSv}$; $\mathrm{P}=0.98)$. In CTA group, $19 \%$ of males $(15 / 79)$ but only $3.4 \%$ of females $(3 / 88)$ had obstructive CAD and required subsequent ICA; $P<0.001)$.

Limitations: The study was performed at a single center in a small number of patients.

Conclusion: This study shows that females equally to males benefit by a reduced length of stay through initially performing CTA instead of ICA Radiation doses were similar in the CTA and ICA group for females and males while the need for subsequent ICA after CTA was significantly lower for females compared to males.

Funding for this study: Grant support from the German Research Foundation through the Heisenberg Programme.

Author Disclosures:

K. Kofoed: Board Member; steering committee of the CORE320 trial. Equipment Support Recipient; Toshiba Medical Corporation. Investigator; Principle investigator of the investigator initiated CATCH-2 trial, CSub320 trial. Research/Grant Support; AP Møller og hustru Chastine McKinney Møllers Fond, The John and Birthe Meyer Foundation, Research Council of Rigshopitalet, The University of Copenhagen, The Danish Heart Foundation, The Lundbeck Foundation, The Danish Agency for Science, Technology and Innovation by The Danish Council for Strategic Research. Speaker; Speakers Bureau of Toshiba Medical Systems. M. Dewey: Research/Grant Support; Heisenberg Program of the DFG for a professorship (DE 1361/14-1), Digital Health Accelerator of the Berlin Institute of Health, the FP7 Program of the European Commission for the randomized multicenter DISCHARGE trial (603266-2, HEALTH-2012.2.4.-2), European Regional Development Fund (20072013 2/05, 20072013 2/48), German Heart Foundation/German Foundation of Heart Research (F/23/08, F/27/10), Joint Program from the German Research Foundation (DFG) and the German Federal Ministry of Education and Research (BMBF) for meta-analyses (01KG1013, 01KG1110, 01KG1210). Speaker; Toshiba Medical Systems, Guerbet, Cardiac MR Academy Berlin, Bayer (Schering-Berlex). Other; editor of Coronary CT Angiography and Cardiac CTboth published by Springer, Institutional master research agreements exist with Siemens Medical Solutions, Philips Medical Systems, and Toshiba Medical Systems.

$11: 10$

Discussant

R. Marano; Rome/IT 


\section{B-0598 11:15}

Effect of coronary computed tomography vs invasive coronary angiography on statin adherence and serum lipid levels in patients with atypical chest pain: a randomised controlled trial

L. Elzenbeck ${ }^{1}$, S. Feger ${ }^{1}$, P. Martus ${ }^{2}$, N. Rieckmann ${ }^{1}$, K. Stangl $^{1}$, A. Marek ${ }^{1}$, H. Dreger ${ }^{1}$, M. Beling ${ }^{1}$, E. Zimmermann ${ }^{1}$, M. Rief ${ }^{1}$, B. Chow ${ }^{3}$,

P. Maurovich- Horvat ${ }^{4}$, M. Laule ${ }^{1}$, M. Dewey ${ }^{1} ;{ }^{1}$ Berlin/DE, ${ }^{2}$ Tübingen/DE,

${ }^{3}$ Ottawa, BC/CA, ${ }^{4}$ Budapest/HU (laura.elzenbeck@charite.de)

Purpose: To determine whether coronary computed tomography angiography (CTA) compared with invasive coronary angiography (ICA) improves statin therapy adherence and long-term serum lipids.

Ethics committee approval: By ethics committee (EA-1-080-08) and Federal Office for Radiation Protection (Z5-22462/2-2008-048). Written informed consent was given before randomisation.

Methods and Materials: Single-center randomised trial with blinded observers. 340 patients with a clinical indication for ICA and atypical chest pain were randomised to CTA or ICA. Statin recommendations were based on European guidelines. Statin intake and serum lipids were compared.

Results: Baseline lipids were available for 164 of 167 (98\%) and 160 of 162 $(99 \%)$ patients who underwent CTA and ICA, respectively. Follow-up was available for 155 of 164 (95\%) and 141 of 160 patients (88\%) leaving 296 $(90 \%)$ for analysis. Approximately one in four patients were on statin therapy at baseline (CTA: 37/155 [24\%]; ICA: 32/141 [23\%]; P=0.46). Statins were recommended significantly more often to patients without baseline statins in the CTA group (65\% [77/118] vs. $47 \%$ [51/109]; $P=0.007)$. Adherence at followup after 3.3 years (IQR=1.3-4.6) was greater in the CTA group (60\% [46/77] vs $39 \%$ [20/51]; $P=0.03)$. At long-term follow-up, total cholesterol and low-density lipoprotein improved in the CTA group $(-5.6 \pm 22.6 \%,-4.3 \pm 35.5 \%)$ but increased in the ICA group $(+2.4 \pm 19.8 \%,+4.6 \pm 28.9 \% ; P<0.001, P=0.019)$.

Limitations: Longer follow-up would be needed to investigate cardiovascular events.

Conclusion: Coronary CTA resulted in greater adherence to statin recommendations and improved serum lipids after 3.3 years.

Funding for this study: Grant support through the Heisenberg Programme.

$11: 25$

Discussant

R. Vliegenthart; Groningen/NL

\section{B-0599 11:30}

The role of intraprostatic antibiotic injection for reducing infectious complications of transrectal ultrasound-guided biopsy

F. Shobeirian, M. Darabi, S.M. Bagheri, M. Zare Mehrjardi, A. Rezayi; Tehran/IR (farzaneh.shobeirian@gmail.com)

Purpose: The overall risk of trans-rectal prostate biopsy is low, but it is associated with significant morbidity. In this study, a method for reducing infectious complications of this procedure is investigated.

Ethics committee approval: Our clinical trial has been approved by Ethics Committee. All patients signed a written informed consent.

Methods and Materials: In this randomised clinical trial, we prescribed the prophylactic oral antibiotic (Ciprofloxacin, $500 \mathrm{mg}$ once daily) one day before performing the prostate needle biopsy, until two days after the procedure in 210 patients with 40-70-year-old age who were candidates for TRUS-guided prostate biopsy. The enrolled patients were randomly allocated to two equal groups (105 patients in each one). Systemic oral antibiotic and topical disinfection of the rectum by povidone-iodine $1 \%$ solution were applied for all patients, while direct intraprostatic injection of antibiotic (Amikacin, $1 \mathrm{ml}$ into each prostate lobe) was performed just in one of the groups. All of the patients were followed for 30 days for the development of any symptoms of infection including fever, pelvic pain, and pyuria.

Results: 210 patients were equally divided into two group. The mean age and prevalence of comorbidities were not significantly different between two groups. Serum PSA level and prostate volume also did not have a significant difference in two groups. Of the patients without intraprostatic injection of Amikacin, 9 cases $(8.6 \%)$ developed infectious complications, including lower urinary tract infection (7 cases) and prostatitis (2 cases). These complications led to hospitalization in 6 patients. None of the patients who received intraprostatic antibiotic during TRUS-guided biopsy developed an infection. The difference between two groups for developing infectious complications was statistically significant ( $p=0.003$ ).

Limitations: A multi-centric study with a larger sample volume could provide more accurate results.

Conclusion: Local prophylactic antibiotic injection during sonography guided trans-rectal prostate biopsy can significantly reduce the rate of infectious complications.

Funding for this study: There was no funding from industry or a funding agency.
11:39

Discussant

R.H. Oyen; Leuven/BE 


\section{Friday, March 2}

\section{Breast}

\section{CT 10}

\section{Clinical Trials in Radiology 3}

Moderators:

M. Dewey; Berlin/DE

R.L. Ehman; Rochester, MN/US

\section{B-1006 10:30}

Breast cancer screening with tomosynthesis: distribution of cancer subtypes in the Malmö Breast Tomosynthesis Screening Trial K.S. Johnson ${ }^{1}$, S. Zackrisson ${ }^{1}$, A. Rosso ${ }^{1}$, H. Sartor ${ }^{1}$, I. Andersson ${ }^{1}$, L.H. Saal ${ }^{2}$,

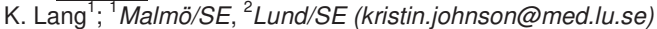

Purpose: Digital breast tomosynthesis (DBT) has the potential of becoming the new breast cancer screening modality. It is, however, not known if DBT screening will change the types of cancers detected. We analysed the tumour biology of invasive breast cancers detected in the Malmö Breast Tomosynthesis Screening Trial (MBTST), using the St. Gallen surrogate subtype classification, clinically used for prognostic and treatment guidance. Ethics committee approval: The study was approved by the Regional Ethical Review Board at Lund University (Dnr 2009/770). Written informed consent was obtained.

Methods and Materials: The MBTST is a prospective, population-based onearm screening trial comparing one-view DBT with two-view digital mammography (DM). 14848 women were enrolled and in total 118 invasive cancers were detected. The cancers were classified according to St. Gallen; luminal A-like, luminal B-like HER2-/HER2+, HER2+ and triple negative. The subtypes were presented by mode of detection; DBT alone compared to all DM detected cancers.

Results: In total, 38 breast cancers were detected with DBT alone (37 with subtypes): 19 (50\%) luminal A-like, 14 (37\%) luminal B-like HER2-, 0 (0\%) luminal B-like HER2+, $1(3 \%)$ HER2+ and $3(8 \%)$ triple negative 80 cancers were detected with both DBT and DM $(n=76)$ or DM alone $(n=4): 37(46 \%)$ luminal A-like, $34(42 \%)$ luminal B-like HER2-, 1 (1\%) luminal B-like HER2+, 3 (4\%) HER2+ and $5(6 \%)$ triple negative.

Limitations: The study was performed on an urban Swedish population using one particular brand of tomosynthesis equipment and might, therefore, not be generalizable to other populations and to the use of other tomosynthesis vendors.

Conclusion: Invasive cancers detected with DBT alone or DM in the MBTST were of similar subtypes according to the St. Gallen classification indicating that DBT screening may not alter the treatment predictive and prognostic panorama of screen-detected cancers.

Funding for this study: Swedish Research Council, Swedish Cancer Society, Skane county council's research and development foundation, Medical Faculty at Lund University (ALF grant), Unilabs Sweden and Siemens Healthineers. Author Disclosures:

S. Zackrisson: Speaker; Siemens. H. Sartor: Speaker; Siemens. K. Lang: Speaker; Siemens.

10:40

Discussant

F.J. Gilbert; Cambridge/UK

\section{B-1007 10:45}

Digital breast tomosynthesis vs digital mammography: recallrate by mammographic density, interim analyses

H. Aase ${ }^{1}, \AA$ A.S. Holen ${ }^{2}$, B. Hanestad ${ }^{1}$, S. Hofvind ${ }^{2} ;{ }^{1}$ Bergen/NO, ${ }^{2}$ Oslo/NO (hildegunn.aase@helse-bergen.no)

Purpose: To compare recall rates using synthetic mammography + digital breast tomosynthesis (SM+DBT) versus digital mammography (DM) in the Norwegian Breast Cancer Screening Program, stratified by mammographic density.

Ethics committee approval: The study is approved by the Regional committees for medical and health research ethics (2015/424) and registered in Clinical trials.org (NCT02835625).

Methods and Materials: As part of a randomised controlled screening trial performed in Bergen, 7037 women were screened with SM+DBT and 7052 with DM, January-December 2016. We obtained continuous measures of volumetric breast density (VBD) using an automated software (Volpara version 1.5.1). Recall rate for positive mammographic findings was calculated for women who underwent screening with the two techniques, stratified by quartiles of VBD; $(1:<3.5 \% ; 2: 3.5-5.4 \% ; 3: 5.4-8.8 \%$ and $4:>8.8 \%)$. Twoproportion z-tests was used to test for statistical significance between the groups $(p<0.05)$.

Results: Recall rate was statistically significantly lower for SM+DBT $(3.0 \%$ [208/7037]) compared to DM (3.6\% [254/7052], $\mathrm{p}=0.03)$. The recall rate increased by mammographic density for SM+DBT, from $2.1 \%$ for VBD-1 to $4.5 \%$ for VBD-4. For DM, the recall rate remained the same for different quartiles of mammographic density. For DM, the highest recall rate $(4.3 \%)$ was observed for VBD-3.

Limitations: The radiologists had limited experience with DBT when the trial started.

Conclusion: Women screened with SM+DBT had a lower recall rate compared to those screened with DM. The higher recall rate among women with dense breast and screened with SM+DBT need to be followed closely according to cancer detection and rate of false positive screening results.

Funding for this study: The project is funded by the Norwegian Research Council.

10:55

Discussant

E.M. Fallenberg; Berlin/DE

\section{B-1008 11:00}

Reduction in infarct volume on CT and functional outcome after endovascular treatment for acute ischaemic stroke: a causal mediation analysis

K. Compagne ${ }^{1}$, M. Boers ${ }^{2}$, H. Marquering ${ }^{2}$, C.B. Majoie ${ }^{2}$, W. Van Zwam $^{3}$ D.W. Dippel ${ }^{1}$, A. van Es ${ }^{1}$, A. Van der Lugt ${ }^{1}$, H. Lingsma ${ }^{1} ;{ }^{1}$ Rotterdam/NL

${ }^{2}$ Amsterdam/NL, ${ }^{3}$ Maastricht/NL (c.compagne@erasmusmc.nl)

Purpose: A potential explanation for the favourable effect of endovascular treatment (EVT) on functional outcome after acute ischaemic stroke is a reduction in ischaemic lesion volume (ILV). ILV is strongly related to the functional outcome. The aim of the present study was to assess to which extent ILV also explains the effect of EVT on functional outcome.

Ethics committee approval: The study protocol of the MR CLEAN trial was approved by a central medical ethics committee and the research board of each participating center. All patients or their legal representatives provided written informed consent before randomisation.

Methods and Materials: The data of all 500 patients included in the randomised controlled MR CLEAN trial on the effectiveness of EVT were evaluated. ILV was assessed on non-contrast CT scan 5 days after stroke. The functional outcome was the score on the modified Rankin Scale at three months. We tested the pathway from EVT via ILV to functional outcome with a mediation model, using ordinal regression, with adjustment for relevant baseline covariates.

Results: In total, 452 patients were analysed. EVT patients had better outcome (acOR 2.01 $(95 \% \mathrm{Cl} 1.43-2.82)$. EVT patients had smaller median ILV $(55 \mathrm{~mL})$ than non-EVT patients $(80 \mathrm{~mL}$ ) (absolute difference $25 \mathrm{~mL} ; 95 \% \mathrm{C}$ 17.26-44.92). ILV was inversely associated with better outcome (common odds ratio (cOR) per $10 \mathrm{~mL} 0.58 ; 95 \% \mathrm{Cl} 0.51-0.66$ ). After adjustment for ILV, the effect of EVT on the outcome decreased, but remained substantial (acOR 1.80; $95 \% \mathrm{Cl} 1.28-2.55)$. Reduction of ILV explained $16 \%$ of the beneficial effect of EVT on outcome.

Limitations: A limitation of our study is the exclusion of deceased patients in the first week after onset and therefore missing FIV measurements at 5-7 day follow-up which is tried to overcome by imputation.

Conclusion: The reduction of ILV by EVT explains only part of the effect on functional outcome. Before ILV is used as a primary outcome in efficacy trials, improvement of this potentially valuable outcome measure is needed.

Funding for this study: The MR CLEAN trial was partly funded by the Dutch Heart Foundation and by unrestricted grants from AngioCare BV, Medtronic/Covidien/EV3®, MEDAC Gmbh/LAMEPRO, Penumbra Inc. Stryker $\AA^{B}$, and Top Medical/Concentric. The MR CLEAN is registered under number NTR1804 in the Dutch trial register and under ISRCTN10888758 in the ISRCTN register. Erasmus Medical Center Rotterdam received funds from Stryker ${ }^{\circledR}$ for consultations by AvdL, AvE and DD and from Bracco Imaging ${ }^{\circledR}$ for consultations by DD. Academic Medical Center Amsterdam received funds from Stryker ${ }^{\circledR}$ for consultations by $C M, Y R$ and $O B, A B$ and $H M$ own stock in Nico-lab B.V. Maastricht University Medical Center received funds from Stryker ${ }^{\circledR}$ and Cerenovus ${ }^{\circledR}$ for consultations by WZ. AY reports research grants form Penumbra Inc. and Neuravi Inc., and consultant fees from Cerenovus/J\&J. No other personal disclosures are reported. Author Disclosures:

C.B. Majoie: Research/Grant Support; Stryker. W. Van Zwam: Research/Grant Support; Stryker, Codman. D.W. Dippel: Research/Grant Support; Stryker, Bracco Imaging. A. Van der Lugt: Research/Grant Support; Stryker. 
$11: 10$

Discussant

I. Szikora; Budapest/HU

\section{B-1009 11:15}

Radiologic follow-up of the NU-AGE trial: body composition changes detected by dual-energy $x$-ray absorptiometry show a correlation with laboratory markers of inflammation

D. Mercatelli ${ }^{1}$, A. Santoro ${ }^{1}$, G. Guidarelli ${ }^{1}$, C. Fabbri ${ }^{1}$, R. Ostan ${ }^{1}$,

A. Berendsen ${ }^{2}$, B. Pietruszka ${ }^{3}$, A. jennings ${ }^{4}$, N. Meunier ${ }^{5}$, M. Aparisi Gomez ${ }^{6}$

E. Caumon ${ }^{1}$, S. Fairweather-Tait ${ }^{4}$, A. Bialecka ${ }^{3}$, C. de Groot ${ }^{7}$, G. Guglielmi ${ }^{8}$,

G. Battista ${ }^{1}$, C. franceschi ${ }^{1}$, A. Bazzocchi ${ }^{1} ;{ }^{1}$ Bologna/IT, ${ }^{2}$ Wageningen/NL,

${ }^{3}$ Warsaw/PL, ${ }^{4}$ Norwich/UK, ${ }^{5}$ Clermont-Ferrand/FR, ${ }^{6}$ Auckland/NZ

${ }^{7}$ Wageningen $/ I T,{ }^{8}$ Andria/lT (abazzo@inwind.it)

Purpose: To investigate changes in body composition(BC) parameters detected by dual-energy X-ray absorptiometry(DXA) in an elderly population following a one-year dietary intervention to build a predictive model of risk integrating omics data. The specific aim of this work was to investigate the degree of correlation between changes in BC and plasma levels of inflammatory markers.

Ethics committee approval: The 5 centres involved in the study obtained protocol approval by local Ethics committees. Informed consent was obtained from research participants at baseline.

Methods and Materials: A total of 1295 volunteers free of major overt diseases, aged 65 to 79, were enrolled in 5 European countries(Italy, France, United Kingdom, Netherlands, and Poland). Interview, anthropometric measurements, whole-body DXA scans, blood, urine and faeces samples were taken at baseline/T1. Comparison of variables was performed using Wilcoxon rank-sum test. Correlation analysis was performed using Spearman rank test. Results: DXA follow-up analysis revealed a significant decrease of fat mass(FM), fat mass/lean mass (FM/LM) and android FM/LM in the diet group of the Italian cohort. Plasma level changes of pro-inflammatory marker leptin were positively correlated with changes in $\mathrm{FM}$ ( $\mathrm{rho}=0.179, \mathrm{p}=0.048), \mathrm{LM}$ (rho=0.201, $p=0.026$ ) andandroid/gynoid FM (rho=0.193, $p=0.033$ ), while the decrease of android FM/LM was negatively correlated with $\mathrm{C}$-reactive protein plasma levels $(\mathrm{rho}=-0.433, \mathrm{p}=0.0189$ )

Limitations: DXA scans were performed with different devices in the 5 centres.

Conclusion: BC markers may beuseful to predicta specific inflammatory risk profile in an elderly population during follow-up.

Funding for this study: This project was supported by the European Union's Seventh Framework Program.

$11: 25$

Discussant

F. Bamberg; Tübingen/DE

\section{B-1010 11:30}

An international randomised controlled trial of two interventions for reducing doses for computed tomography through audit feedback and sharing best practices

R. Smith-Bindman ${ }^{1}$, P. Chu ${ }^{1}$, Y. Wang ${ }^{1}$, R. Chung ${ }^{1}$, A. Einstein ${ }^{2}$, D. Miglioretti ${ }^{3}$. ${ }^{1}$ San Francisco, CA/US, ${ }^{2}$ New York, NY/US, ${ }^{3}$ Davis, CA/US

(rebecca.smith-bindman@ucsf.edu)

Purpose: Few interventions for reducing radiation doses from CT have been studied.

Ethics committee approval: Approvals received.

Methods and Materials: We evaluated two interventions to optimize CT radiation effective dose using a randomized trial: a) simple audit comparing institutional doses with tailored recommendations; b) multicomponent intervention with audit plus 8-week educational course. We included 1,149,671 CT examinations from 103 institutions ( 6 countries) between November 2015 and August 2017. Mean effective dose and proportion of high-dose examinations within anatomic areas following each intervention were compared to baseline using hierarchical generalized linear models adjusting for temporal trends.

Results: Following the multicomponent intervention, mean dose and proportion of high dose examinations decreased significantly for all examination types $(p<0.0001)$ : abdomen examinations $=2.7 \mathrm{mSv}(19 \%)$ reduction in mean effective dose, $45 \%$ reduction in high-dose examinations; chest $=0.9 \mathrm{mSv}(11 \%)$ reduction in mean effective dose, $30 \%$ reduction of highdose examinations; combined chest and abdomen $=3.7 \mathrm{mSv},(20 \%)$ reduction in mean effective dose; $57 \%$ reduction of high-dose examinations; head $=0.10$ mSv $(5 \%)$ reduction in mean effective dose, $19 \%$ reduction in high dose examinations. The simple audit also significantly reduced dose $(\mathrm{p}<0.05)$; however, the magnitudes were smaller than for the multicomponent intervention. Both interventions were more effective when combined with a 2day in-person educational meeting.
Limitations: All institutions used dose monitoring software demonstrating an interest in dose optimisation.

Conclusion: Providing detailed feedback to institutions on their CT doses combined with actionable suggestions and education results in significant reductions in average CT doses and proportion of high-dose examinations. Funding for this study: $\mathrm{NIH}$

Author Disclosures:

R. Smith-Bindman: Advisory Board; Bayer. Research/Grant Support; National Institutes of Health; Patient Centered Outcomes Research Institute.

$11: 40$

Discussant

A. Trianni; Udine/IT

\section{B-1011 11:45}

Prophylactic hydration to prevent contrast-induced nephropathy (AMACING): long-term results of a prospective, randomised, controlled, non-inferiority trial

E.C. Nijssen; Maastricht/NL (estelle.nijssen@mumc.nl)

Purpose: The AMACING trial evaluated the cost and clinical effectiveness of current clinical practice guidelines on intravascular iodinated contrast materia use. The study population included $>90 \%$ patients marked as high risk by the guidelines. The results showed no prophylaxis to be non-inferior to standard prophylactic intravenous hydration in the prevention of contrast-induced nephropathy $(\mathrm{CIN})$. The current paper presents the long-term data of the AMACING trial.

Ethics committee approval: The Maastricht University Medical Centre research ethics committee approved the study before first inclusion.

Methods and Materials: AMACING is a prospective, randomised, parallelgroup, open-label, non-inferiority trial of patients at risk of CIN according to current guidelines, with eGFR30-59 $\mathrm{mL} / \mathrm{min} / 1 \cdot 73 \mathrm{~m}^{2}$ and undergoing elective procedure requiring iodinated contrast material administration. Between June 17,2014 , and July 17, 2016, 660 consecutive patients were randomly assigned $(1: 1)$ to receive no prophylaxis $(n=332)$ or standard intravenous hydration with normal saline according to the guidelines $(n=328)$. We excluded patients with eGFR<30 mL/min/1.73 $\mathrm{m}^{2}$, previous dialysis, or no referral for intravenous hydration. Randomisation was stratified by predefined risk factors. Incidence of dialysis, mortality and renal function were registered up to 1 year post-contrast exposure. Subgroup analyses were performed based on pre-defined stratification risk factors. ClinicalTrials.gov, NCT02106234.

Results: Dialysis was recorded in $2 / 332(0.60 \%)$ non-hydrated patients and in $2 / 328(0.61 \%)$ hydrated patients. Absolute difference (no hydration vs hydration) $-0.01 \%(95 \% \mathrm{Cl}-1.19$ to $1.18 ; \mathrm{p}=0.9909)$. $36 / 332(10.84 \%)$ nonhydrated patients and $32 / 328(9.76 \%)$ hydrated patients died within 365 days. Absolute difference (no hydration vs hydration) $+1.01 \%(95 \% \mathrm{Cl}-3.55$ to 5.72 ; $\mathrm{p}=0.6490)$.

Limitations: See Lancet 2017; 389:1312-22.

Conclusion: Assuming optimal contrast media administration, withholding prophylaxis for high-risk patients with eGFR>29 mL/min/1.73 $\mathrm{m}^{2}$ might be considered without compromising patient safety.

Funding for this study: Stichting de Weijerhorst

$11: 55$

Discussant

P. Aspelin; Stockholm/SE 